\title{
Characteristics of HOMO and LUMO Potentials by Altering Substituents: Computational and Electrochemical Determination
}

\author{
Young-Sung Kim, Sung-Hoon Kim¹, Tae-Kyung Kim ${ }^{1}$ and Young-A Son ${ }^{\dagger}$ \\ BK 21 FTIT, Dept. of Organic Materials and Textile System Engineering, \\ Chungnam National University, Daejeon, 305-764, South Korea \\ ${ }^{1}$ Dept. of Textile System Engineering, Kyungpook National University, Daegu, 702-701, South Korea
}

(Received: September 5, 2008/Revised: October 16, 2008/Accepted: October 21, 2008)

\begin{abstract}
Recently, computational calculation of molecular energy potentials and electrochemical reduction/oxidation behaviors are of very importance in view point of prediction of dye's properties such as energy levels and bandgaps of absorption. This can be influenced by their different constituents or substituents in chromogen molecules. Structural conformations and properties with computational modeling calculation are numerically simulated, which are fully or partly based on fundamental laws of physics. In addition, cyclic voltammetric measurement was used to obtain the experimental redox potential values, which were compared to the computed simulation values.
\end{abstract}

Keywords: cyclic voltammetry, HOMO, LUMO, computational calculation, antraquinone dye, molecular modeling

\section{Introduction}

Anthraquinone and azo dyes have been the most widely used class of organic dyes. Two dye types, as the largest group of colorants and historically the most important categories, have been widely applied in chemistry, biochemistry and textile industry, especially as useful coloration dye stuff ${ }^{1-4)}$. In addition, nowadays, these synthetic derivatives of anthraquinone and azo dyes $^{4-6)}$ have been enjoyed for other purposes as anticancer drug for anthraquinone and microfluidic dye synthesis for azo types. However, the widely used main target is the textile dyeing industries.

In this work, the effects of the chemical structures of six dyes were chosen with relation to the altering characteristics of dye substituents. Anthraquinone and azo dyes having different substituents were examined to determine their potential energy levels in ground and excited states, namely HOMO and LUMO.

All theoretically computational calculations for the energy potential and the electron distribution of HOMO /LUMO states were performed by means Material Studio 4.3 package program ${ }^{7-13)}$.
HOMO/LUMO energy levels were concerned with the states of molecular orbital and the identifications of electron transition ${ }^{13,14}$. Thus, this theory is quite useful for the application of electron chemistry, because HOMO /LUMO potentials involve the functions of proton transfer or unshared pair/empty orbital interaction.

Futhermore, we have also considered cyclic voltammetric (CV) experiments to determine the energy potentials for the electrochemical reduction/oxidation potential of anthraquinone and azo dyes. These determined energy levels by electrochemical reduction were compared to the values of computational calculated ones. The effect of the structural properties of the dye molecules was studied by qualitative structure- zelectrochemistry relationship analysis.

All dyes were obtained from commercial sources. The dyes used were listed in Table 1 . The computational calculation was used with Material studio 4.3.

The geometrical optimization and energy potential of HOMO/LUMO states were stabilized and calculated.

Electrochemical measurement was used by cyclic voltammetry (CV) electrode system ustilizing a Versa

Corresponding author. Tel: +82-42-821-6620; Fax: +82-42-823-3736; e-mail: yason@cnu.ac.kr 
STAT3 model. Cyclic voltammery experiment was run in an acetonitrile solution containing tetrabutlyammonium hexafluorophosphate elelctrolyte. The reference electrode, $\mathrm{Ag} / \mathrm{Ag}^{+}$was directly immersed in the reaction cell. The working electrode was a glassy carbon. The counter electrode was a platinum wire. The scan rate was commonly $100 \mathrm{mV} / \mathrm{s}$. HPLC grade acetonitrile was used as purchased and used in the electrochemical redox potential measurements of all six dyes.

For the used dyes, namely three anthraquinone and azo dyes, respectively, the difference of HOMO/LUMO energy level was calculated using computational technique and measured using electrochemical cyclic voltammetric method. The obtained energy potentials of HOMO/LUMO states were compared. The geometrical conformation of molecular structures and electron density distributions were also computationally calculated.

These characteristics were useful to understand the electron distribution through the molecules in HOMO and LUMO energy potentials.

Fig. 1 and Fig. 2 show the computational calculations to expect the HOMO/LUMO energy potentials of anthraquinone and azo dyes. To obtain more detailed insight and information towards the intramolecular charge transfer process of six dyes, the computational theoretical calculation was carried out using DMol3 program in the Material Studio 4.3 package ${ }^{15,16)}$. Fig. 1 and Fig. 2 represent the electron distribution of HOMO/ LUMO energy potential of anthraquinone and azo dyes, respectively. The potentials of HOMO/LUMO energy level of six dyes were computed and listed (Table 2 and Table 3). These results by theoretical calculation are in agreement with the obtained data using electrochemical redox measurement technique. Similar changing patterns of HOMO/LUMO energy levels were observed.

Comparison of the electron distribution in the frontier MOs displays the HOMO-LUMO excitation. Electron distribution for the anthraquinone dyes moved the central quinone ring and electron donating group substituted benzene ring at 1,4 position in HOMO state to the whole anthraquinone molecules in LUMO state. Three dyes showed the similar patterns for electron distribution. In the case of three azo dyes, similarly, The rich electron distribution was detected at the part of molecules, where electron donating amine groups are attached. However, these electrons were moved to the electron withdrawing nitro groups in LUMO state for three azo dyes.

In addition, the electrochemical reduction/oxidation behaviors of anthraquinone and azo dyes were determined by cyclic voltammetry $(\mathrm{CV})^{17}$ in dry $\mathrm{CH}_{3} \mathrm{CN}$.

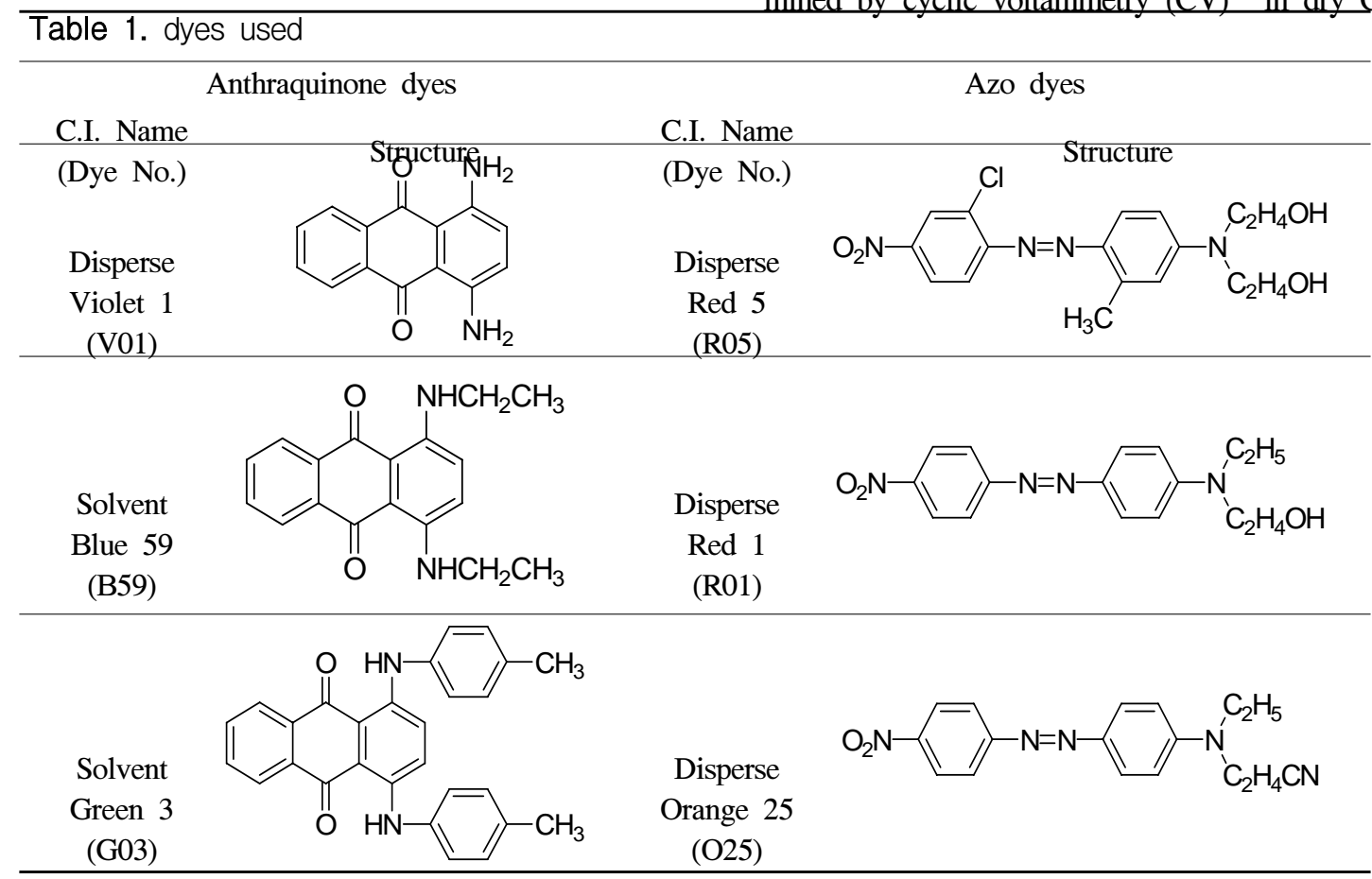



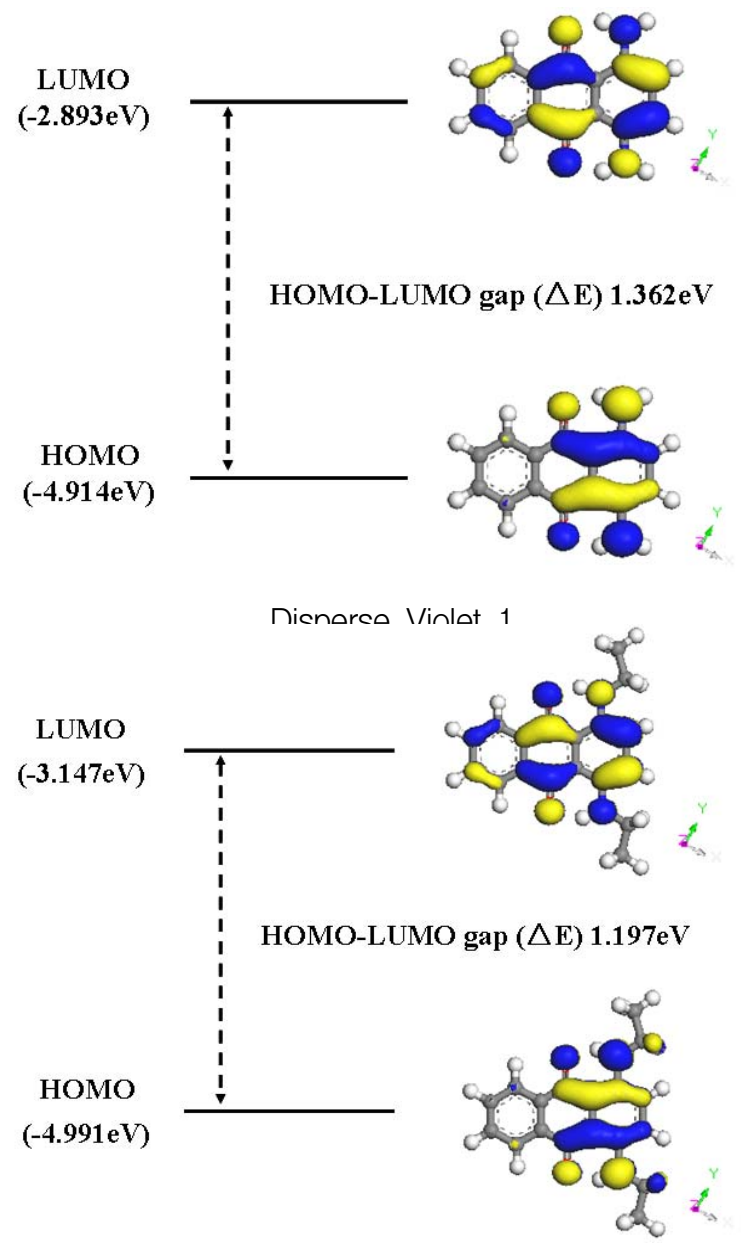

LUMO

$(-3.162 \mathrm{eV})$

HOMO

$(-5.031 \mathrm{eV})$

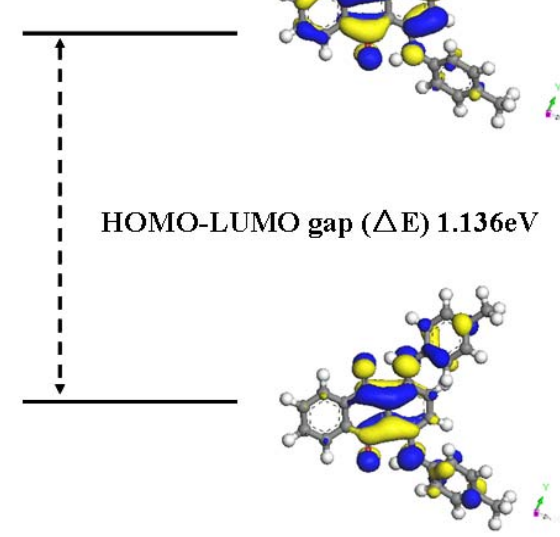

Solvent Green 3

Fig. 1. Electron distribution of $\mathrm{HOMO} / \mathrm{LUMO}$ energy potential of anthraquinone dyes.

Fig. 3 shows the cyclic voltammogram of six dyes. Commonly, the HOMO energy level of these dye molecules can be estimated from its onset oxidation

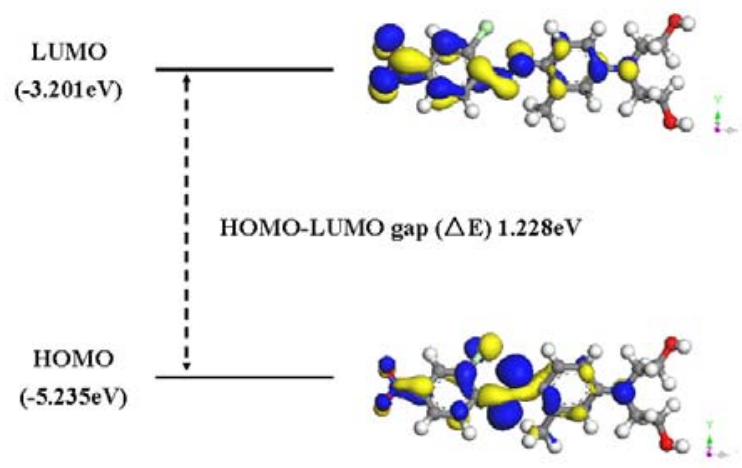

LUMO
$(-2.904 \mathrm{eV})$

Disnerse Red 5

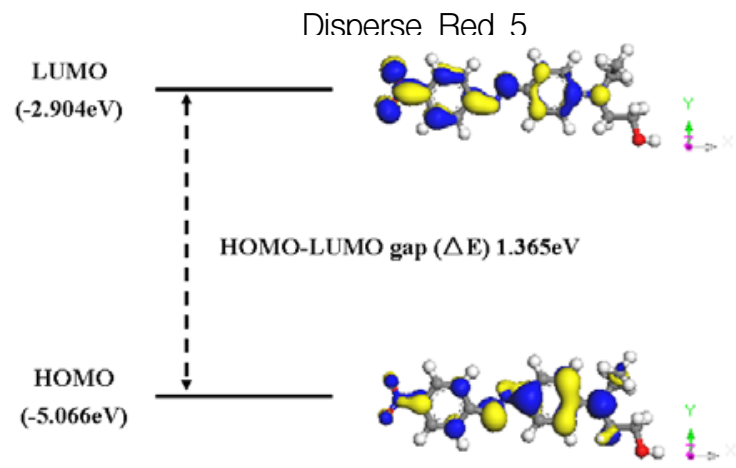

LUMO
$(-2.914 \mathrm{eV})$

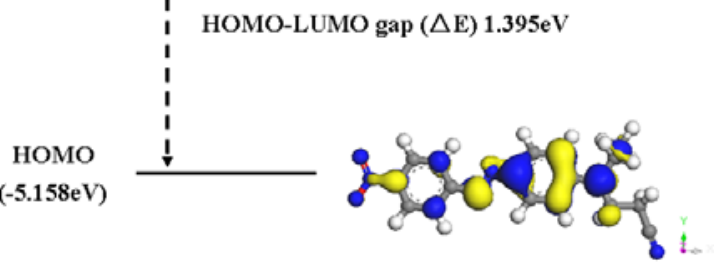

Disperse Orange 25

Fig. 2. Electron distribution of $\mathrm{HOMO} / \mathrm{LUMO}$ energy potential of anthraquinone dyes.

potentials versus a saturated calomel electrode by adding 4.74 and LUMO energy level can be calculated by subtracting its optical bandgap from the HOMO energy level ${ }^{18,19)}$, while the optical bandgap is deduced from the absorption edge.

In addition, the potentials of the highest oxidation peak and the lowest reduction peak can be used to calculate the HOMO/LUMO energy levels.

HOThe (followingQequation=can be used at this method.

$$
-4.8-\left(E_{\text {peak }}-E_{1 / 2}(\text { Ferrocene })\right.
$$


Anthraquinone dyes

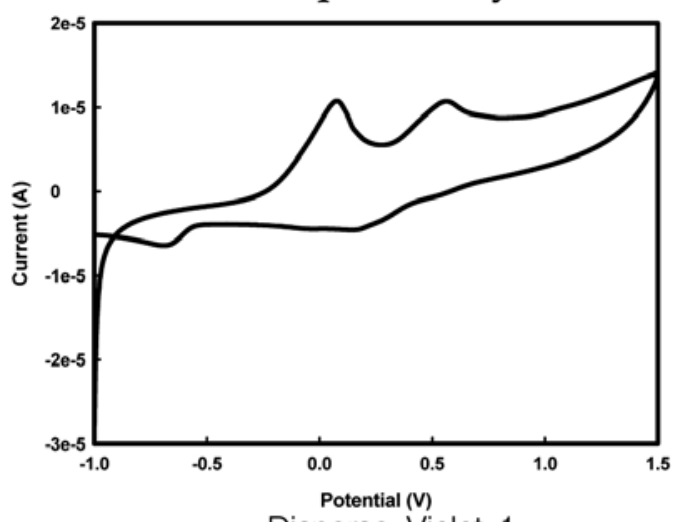

Disperse Violet 1

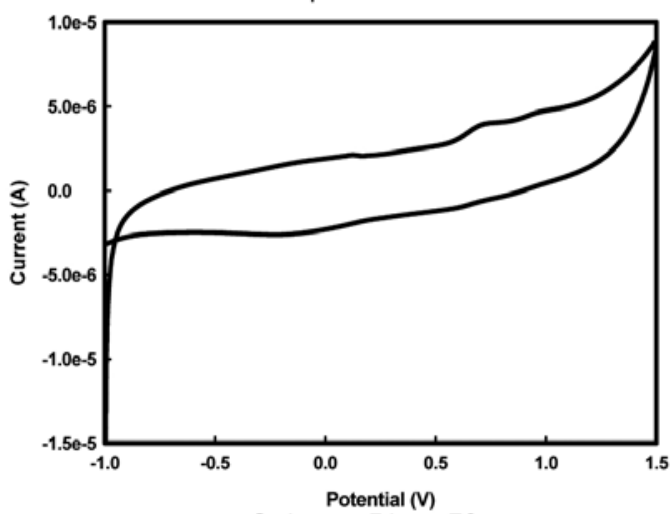

Solvent Blue 59

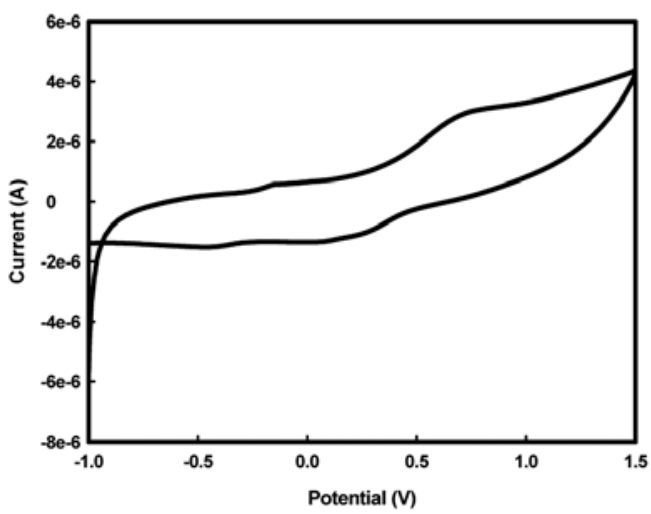

Solvent Green 3
Azo dyes

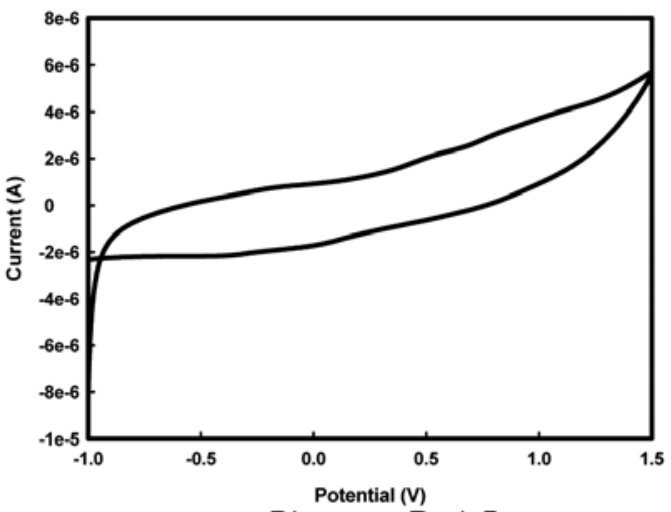

Disperse Red 5

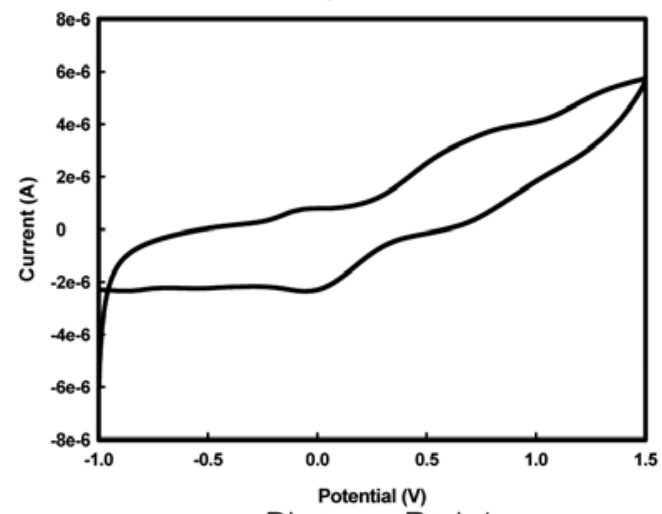

Disperse Red 1

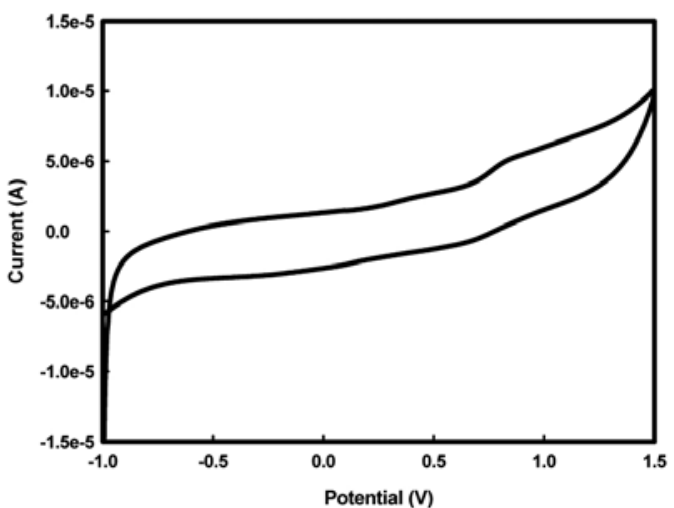

Disperse Orange 25

Fig. 3. Cyclic voltammograms of six dyes used.

Table 2. HOMO/LUMO and bandgap energy potential for anthraquinone dyes

Anthraquinone dyes

$$
\text { Computational calculation Cyclic Voltammetry }
$$

\begin{tabular}{ccccccc}
\hline Anthraquinone dyes & HOMO & LUMO & $\triangle \mathrm{E}$ & HOMO & LUMO & $\triangle \mathrm{E}$ \\
Disperse Violet 1 & $-4.866 \mathrm{eV}$ & $-3.504 \mathrm{eV}$ & $1.362 \mathrm{eV}$ & $-4.914 \mathrm{eV}$ & $-2.893 \mathrm{eV}$ & $2.021 \mathrm{eV}$ \\
Solvent Blue 59 & $-4.742 \mathrm{eV}$ & $-3.545 \mathrm{eV}$ & $1.197 \mathrm{eV}$ & $-4.991 \mathrm{eV}$ & $-3.147 \mathrm{eV}$ & $1.884 \mathrm{eV}$ \\
\hline Solvent Green 3 & $-4.776 \mathrm{eV}$ & $-3.640 \mathrm{eV}$ & $1.136 \mathrm{eV}$ & $-5.031 \mathrm{eV}$ & $-3.162 \mathrm{eV}$ & $1.869 \mathrm{eV}$
\end{tabular}

| 44 | www.ksdf.or.kr 
Table 3. HOMO/LUMO and bandgap energy potential for azo dyes

\begin{tabular}{ccccccr}
\multirow{2}{*}{ Azo dyes } & \multicolumn{2}{c}{ Computational calculation } & \multicolumn{2}{c}{ Cyclic Voltammetry } \\
\cline { 2 - 6 } & HOMO & LUMO & $\triangle \mathrm{E}$ & HOMO & LUMO & $\triangle \mathrm{E}$ \\
\hline Disperse Red 5 & $-5.063 \mathrm{eV}$ & $-3.835 \mathrm{eV}$ & $1.228 \mathrm{eV}$ & $-5.235 \mathrm{eV}$ & $-3.201 \mathrm{eV}$ & $2.034 \mathrm{eV}$ \\
Disperse Red 1 & $-5.220 \mathrm{eV}$ & $-3.855 \mathrm{eV}$ & $1.365 \mathrm{eV}$ & $-5.066 \mathrm{eV}$ & $-2.904 \mathrm{eV}$ & $2.162 \mathrm{eV}$ \\
Disperse Orange 25 & $-5.272 \mathrm{eV}$ & $-3.877 \mathrm{eV}$ & $1.395 \mathrm{eV}$ & $-5.158 \mathrm{eV}$ & $-2.914 \mathrm{eV}$ & $2.244 \mathrm{eV}$ \\
\hline
\end{tabular}

Table 2 and Table 3 show the HOMO/LUMO energy levels for the six dyes, where the potential energy values for the computationally calculated levels and the electrochemically determined levels are shown. For each dyes, the potential energy levels showed the similar characteristic patterns from the values of computational calculation and electrochemical measurement.

\section{Acknowledgements}

This research was financially supporting by the Ministry of Education, Science Technology (MEST) and Korea Industrial Technology Foundation (KOTEF) through the Human Resource Training Project for Regional Innovation.

\section{References}

1. F. Nourmohammadian, I. Yavari, B. Mohtat, S. Zia Shafaei, Density functional theory study of 9,10anthraquinone and its structural isomers, Dyes and pigments, 75, 479-482(2007).

2. A. I. M. Koraiem, R. M. Abu EI-Hamd, A. K. Khalafalah, A. S. Hammam, M. A. EI-Maghraby, M. M. Gomaa, Synthesis and properties of some Naphtho(Quinolino)-Quinone Heterocyclic Dimethine Cyanine Dyes, Dyes and pigment, 30, 89-98(1996).

3. T. Itoh, H. Iwayama, S. Iwatsuki, Synthesis of Donor-Acceptor Substituted Quinonemethide Imines for Nonlinear Optical Materials, Dyes and pigment, 27, 9-15(1995).

4. K. Tae Kyung, S. Young A, Affinity of disperse dyes on poly(ethylene terephthalate) in non-aqueous media. Part 2: effect of substituents, Dyes and pigments, 66, 19-25(2005).

5. W. Huang, Structural and computational studies of azo dyes in the hydrazone form having the same pyridine-2,6-dione component (II): C.I. Disperse Yellow 119 and C.I. Disperse Yellow 211, Dyes and pigment, 79, 69-75(2008).

6. O. Gungor, A. Yilmaz, S. Memonc, M. Yilmaz, Evaluation of the performance of calix[8]arene derivatives as liquid phase extraction material for the removal of azo dyes, Dyes and pigment, 158, 202-207(2008).

7. J. N. Latosinska, M. Latosinska, J. Kasprzak, ${ }^{35} \mathrm{Cl}-\mathrm{NQR}$ and DFT study of electronic structure of amlodipine and felodipine vascular-selective drugs from the dihydropyridine $\mathrm{Ca}^{++}$antagonists group, Chemical Physics Letters, 85, 61-69(2008).

8. F. Brovelli, B. L. Rivas, J. C. Bernede, M. A. del Valle, F. R. Diaz, Y. Berredjem, Electrochemical and optical studies of 1,4-diaminoanthraquinone for solar cell applications, Polymer Bulletin, 58, 521527(2007).

9. M. Shamsipur, A. Siroeinejad, B. Hemmateenejad, A. Abbaspour, H. Sharghi, K. Alizadeh, S. Arshadi, Cyclic voltammetric, computational, and quantitative structure-electrochemistry relationship studies of the reduction of several 9,10-anthraquinone derivatives, Journal of electroanalytical chemistry, 600, 345-358 (2007).

10. P. Bhyrappa, M. Sankar, B. Varghese, Mixed Substituted Porphyrins: Structural and Electrochemical Redox Properties, Inorganic chemistry, 45, 41364149(2006).

11. S. Tsutsui, K. Sakamoto, H. Yoshida, A. Kunai, Cyclic voltammetry and theoretical calculations of silyl-substituted 1,4-benzoquinones, Journal of organo metallic chemistry, 690, 1324-1331(2005).

12. W. HuangKamaljit, Structural and computational studies of azo dyes in the hydrazone form having the same pyridine-2,6-dione component (II): C.I. Soc. of Dyers and Finishers, Vol. 20, No. 5 | 45 | 
Disperse Yellow 119 and C.I. Disperse Yellow 211, Dyes and pigment, 79, 69-75(2008).

13. G. B. Ferreira, E. Hollauer, N. M. Comerlato, J. L. Wardell, An experimental and theoretical study of the electronic spectra of tetraethylammonium [bis (1,3-dithiole-2-thione-4,5-dithiolato)M(III)] and tetraethylammonium [bis(1,3-dithiole-2-one-4,5-dithiolato) $\mathrm{M}(\mathrm{III})] \mathrm{M}=\mathrm{Sb}$ or Bi), Spectrochimica Acta Part A, 71, 215-229(2008).

14. C. N. Ramachandran, D. Roy, N. Sathyamurthy, Host-guest interaction in endohedral fullerenes, Chemical Physics Letters, 461, 87 92(2008).

15. B. Delley, An all-electron numerical method for solving the local density functional for polyatomic molecules, J. Chem. Phys., 92, 508(1990).
16. B. Delley, from molecules to solids with the DMOI [sup 3] approach, J. Chem. Phys., 113, 7756-7764 (2000).

17. A. K. Agrawal, S. A. Jenekhe, Electrochemical Properties and Electronic Structures of Conjugated Polyquinolines and Polyanthrazolines, Chem. Mater., 8, 579(1996).

18. R. M. Noyes, Hydrogen Iodide Revisited. Continued Significance Sullivan Experiments, J. Am. Chem. Soc., 96, 7623-7624(1962).

19. C. Q. Ma, L. Q. Zhang, J. H. Zhou, X. S. Wang, B. W. Zhang, Y. Cao, P. Bugnon, M. Schaer, F. Nusch, D. Q. Zhang, Y. Qiu, 1,3-Diphenyl-5-(9phenanthryl)-4,5-dihydro-1H-pyrazole (DPPhP): structure, properties, and application in organic light-emitting diodes, J. Mater. Chem., 12, 3481-3486(2002). 